\title{
Association of Cancer Worry and Physical Activity Behaviors in Adults without Cancer
}

\author{
Jamil M Lane, PhD, MPH \\ Brandon W Qualls, MPA \\ Jason D Freeman, MS \\ Daniel Rodriguez, PhD
}

\begin{abstract}
Background: Cancer worry has been conceptualized as a potential motivator to engage in cancer preventative behaviors like cancer screening, genetic testing, or smoking cessation. It is currently unknown if these findings extend to the domain of physical activity, as physical activity has been associated with decreased cancer risk. Objective: To examine if the association between cancer worry and other health behaviors will extend to physical activity in a sample of adults not diagnosed with cancer. Methods: Data are drawn from the NCI's 2017 Health Information National Trends Survey (HINTS) Iteration 5 Cycle $1(N=2,706)$ dataset, a nationally representative survey of adults in the United States. A KruskalWallis- $H$ test was conducted to determine whether physical activity duration (i.e., daily minutes) differed between cancer worry levels with a post hoc Dunn's multiple comparison test to compare the differences between mean ranks. Results: A Kruskal-Wallis-H test showed statistically significant differences in PA duration (i.e., daily minutes) between groups that differed in their level of cancer worry. PA in minutes on a typical day was significantly lower in those who reported not at all, moderate, and extreme worry about developing cancer compared to those who reported slightly and somewhat worried. Conclusion: Contrary to our expectations, our results suggest that higher cancer worry levels are paradoxically associated with less $P A$. This study's results are significant in that they add to the breadth of literature linking cancer worry to health behaviors and may be used to inform future health promotion interventions.
\end{abstract}

Keywords: Cancer Worry, Physical Activity, Cancer Prevention, Public Health

\section{INTRODUCTION}

Over $25 \%$ of Americans worry to some degree about being diagnosed with cancer, with nearly $10 \%$ of the population experiencing extreme worry (Ferrer et al., 2013; Persoskie et al., 2014; Vrinten et al., 2015). Worry is a cognitive activity that focuses attention on a perceived threat and acts as a facilitator of problem-solving and self-protective behavior to a perceived threat (Davey et al., 1996). Cancer worry is an adverse emotional response to perceived susceptibility to a positive cancer diagnosis (Hay et al., 2006). There is growing evidence that cancer worry may influence intentions to engage in health-promoting behaviors to prevent cancer. Research has shown that people without cancer who spend time thinking about the disease to some extent, may explore useful information to decrease or eradicate feelings of worry (Hay et al., 2006). For example, cancer worry is significantly related to cancer-preventive practices, such as obtaining a cancer screening (Moser et al., 2007; Quaife et al., 2019; Vrinten et al., 2015;) and genetic testing (Lumish et al.,
2017; Palmero et al., 2020), smoking cessation (Janssen et al., 2014; Yong et al., 2014), and surgical procedures like mastectomy to prevent cancer (Hay et al., 2005).

Despite the current literature reporting the influence of cancer worry on positive cancer-protective health behaviors, few studies have explicitly investigated the association between cancer worry and its impact on health-promoting behaviors like physical activity (PA) in a nationally representative sample. A study examining worry, in general, found that individuals who worry more often spend less time being physically active than individuals who worry less often (Clancy et al., 2020). Two studies limited to women showed some evidence that increased cancer worry was associated with increased physical activity levels (Brunet et al., 2014; Bernat et al., 2015). However, a recent review in this area found mixed and limited evidence for an association between worry of cancer recurrence and physical activity in samples of individuals who have previously undergone cancer treatment (Durazo \& Cameron, 2019). There-

Corresponding Author: Jamil M Lane, Department of Counseling and Human Development, University of Rochester, 500 Joseph C. Wilson Blvd, LeChase Hall, Box 270425, Rochester, NY 14627-0425, USA. Email: jlane9@u.rochester.edu. Phone: 484-919-6436 
fore, we seek to clarify the relationship between cancer worry and physical activity and determine if the association between cancer worry and physical activity found in women generalizes to a nationally representative sample.

Additionally, engaging in PA plays an essential role in numerous physiological systems (Brown et al., 2012), ultimately reducing cancer risk. Many studies have shown that high levels of physical activity in adults is associated with lower risk of breast cancer (Eliassen et al., 2010; Kossman et al., 2011; Pizot et al., 2016), bladder cancer (Keimling et al., 2014), kidney cancer (Behrens \& Leitzmann, 2013), colon cancer (Liu et al., 2016), and several other cancers (Moore et al., 2016; Psaltopoulou et al., 2016). Given that cancer worry may positively influence cancer-preventive health behaviors, and the limited literature investigating its contributive role in PA behaviors, it is essential to address this gap. Thus, the objective of this secondary data analysis was to examine if the association between cancer worry and other health behaviors would extend to physical activity in a nationally representative sample of adults.

\section{Design}

METHOD

This was a secondary data analysis of an existing publicly available dataset from the $\mathrm{Na}$ tional Cancer Institute's 2017 Health Information National Trends Survey (HINTS). HINTS is a nationally representative survey of adults (age $\geq 18$ ) designed to collect data on the need to access, use, and understand health-related information (Finney Rutten et al., 2020). The HINTS-5 study was reviewed and approved by the Westat Institutional Review Board (IRB). The study was exempted from IRB review by the NIH Office of Human Subjects.

\section{Participants}

This study's data came from HINTS-5 (Cycle 1) survey responses collected from January 2017 to May 2017 through a mailed questionnaire. A two-stage sampling design consisted of (1) stratification from residential addresses and (2) from sampled households. Locations with high minority subpopulations were oversampled. For this study, data only include respondents $(\mathrm{N}=$ 2,756) who self-reported that they have not been

Table 1. Sample demographic characteristics $(N=2,706)$.

\begin{tabular}{|c|c|c|c|}
\hline & $M(S D)$ & $N$ & $\%$ \\
\hline Age & $52.2(19.7)$ & & \\
\hline \multicolumn{4}{|l|}{ Gender } \\
\hline Male & & 1100 & 40.7 \\
\hline Female & & 1606 & 59.3 \\
\hline \multicolumn{4}{|l|}{ Race/ethnicity } \\
\hline White, non-Hispanic & & 1933 & 75.2 \\
\hline Non-White & & 637 & 24.8 \\
\hline \multicolumn{4}{|l|}{ Education Level } \\
\hline High school or less & & 680 & 25.3 \\
\hline Some college & & 793 & 29.5 \\
\hline Bachelor's degree or more & & 1213 & 45.2 \\
\hline \multicolumn{4}{|l|}{ Household Income } \\
\hline Less than $\$ 20,000$ & & 464 & 18.6 \\
\hline$\$ 20,000$ to $<\$ 35,000$ & & 347 & 13.9 \\
\hline$\$ 35,000$ to $<\$ 50,000$ & & 335 & 13.3 \\
\hline$\$ 50,000$ to $<\$ 75,000$ & & 447 & 17.9 \\
\hline$\$ 75,000$ or more & & 908 & 36.3 \\
\hline
\end{tabular}

* Indicates significant differences between sex $(\mathrm{p}<0.05)$. 
diagnosed with having cancer by answering to the item "Have you ever been diagnosed as having cancer?" From this group, 50 (1.8\%) survey respondents were excluded because of missing data on one or more of the other variables in the current study. Based on these inclusion/exclusion criteria, the study's subsample was $\mathrm{N}=2,706$ survey respondents.

\section{Measures}

To assess cancer worry, respondents were asked: "How worried are you about getting cancer?" This item was assessed on a 5-point Likert scale, with responses including: $1=$ not at all, 2 = slightly, 3 = somewhat, $4=$ moderately, and $5=$ extremely. To measure physical activity durations, respondents were asked to report how long they are typically doing physical activity or exercise of at least moderate intensity in minutes. Participants' self-reported demographic characteristics including race/ethnicity, gender, age, education level, and household income.

\section{Statistical Analysis}

All statistical analyses were conducted using $\mathrm{R}$ program 4.0.3 software with the fisheries stock assessment (FSA; Ogle, 2016) and dplyr (Wickham et al., 2015) packages. Descriptive statistics (means, standard deviations, frequencies, and percentages) were calculated for all variables. We conducted a Kruskal-Wallis-H test to determine whether physical activity duration (i.e., daily minutes) was different for cancer worry levels. A post hoc Dunn's (1964) multiple comparison test compared the differences (mean ranks) between more than two groups. The non-parametric test was used because the study variables were not normally distributed.

\section{RESULTS}

Participants $(\mathrm{N}=2,706)$ were 52 years old on average in Cycle 1 (Table 1). The majority of participants were female (59\%) and White $(75 \%)$ race/ethnicity. With respect to education level, $25.3 \%$ had a high school education or less, $29.5 \%$ attended some college, and $45.2 \%$ had a bachelor's degree or more.

A Kruskal-Wallis $\mathrm{H}$ test was conducted to determine if there were differences in physical activity (PA) duration (i.e., minutes) between groups that differed in their level of cancer worry: not at all, slightly, somewhat, moderately, and extremely worried. Results showed that minutes of PA on a typical day was associated with levels of cancer worry, $x_{(4)}^{2}=13.401, \mathrm{p}<.01$ (Table 2). More specifically, on a typical day, PA in minutes was statistically lower in those not at all, moderately, and extremely worried about developing cancer than among those who were somewhat and slightly worried about developing cancer. Posthoc pairwise comparisons were conducted using a Dunn's (1964) procedure with a Bonferroni correction for multiple comparisons. Results indicated a statistically significant difference in PA in minutes between somewhat and moderately worried, and not at all and somewhat worried, $\mathrm{p}$ $<.05$. There was no statistically significant difference between other worry groups.

\section{DISCUSSION}

The purpose of this study was to examine the relationship between cancer worry and physical activity durations in adults. A Kruskal-Wallis$\mathrm{H}$ test was used to determine whether physical activity durations (i.e., daily minutes) differed by cancer worry levels. Contrary to our expectations, our results suggest that no worry, moderate, and extreme cancer worry levels are linked to less PA, whereas slightly or somewhat cancer worry levels are linked to greater PA.

Lower PA behaviors among no worry and elevated worry individuals observed in this study are consistent with limited research indicating that worry levels about getting cancer can lead to more significant avoidance and lower health-promoting behaviors. For example, very low or no worry levels may induce inactivity in health-promoting behaviors (Consedine et al., 2004a). In contrast, elevated levels of cancer worry strongly predict doctor avoidance (Persoskie et al., 2014), interrupt healthy decision-making (Miles et al., 2008), and can be a barrier for other health behaviors including smoking-cessation intentions (Klein et a., 2009) and cancer screening (Andersen et al., 2003; Consedine et al., 2004a). Evidence also shows that elevated worry levels contribute to low fruit and vegetable intake, less physical activities (Ferrer et al., 2013), longer durations of sitting, and less walking among adults (Clancy et al., 2020). However, our findings are inconsistent with some previous literature indicating that elevated worry could act as an adaptive function by motivating preventive care behaviors. For example, elevated cancer worry significantly predicted the desire to adhere to physical activity guidelines in college women (Bernat et al., 2015). Additionally, elevated levels of worry for developing lung cancer was positively related to greater intentions to quit smoking (Janssen et al., 2014; Yong et al., 2014), while developing breast cancer was positively related to routine mammography (Consedine et al., 2004b) and colorectal cancer was positively related to routine sigmoidoscopy and colonoscopy screening (Moser et al., 2007).

\section{Study strengths and limitations}

The current study has both strengths and limitations. Strengths include reporting results from a large, diverse, and nationally representative sample of adults. Limitations include the use of only one cycle of the HINTS study, and the crosssectional and observational nature of the data, making it difficult to infer causation. Another limitation is the reliability and validity of singleitem cancer worry and PA duration measures, which also have lower sensitivity than alternative measures and were all based on self-reports that are subject to response bias. Lastly, it is unclear 
Lane, Qualls, Freeman, Rodriguez

Table 2: Kruskal Wallis-H test results of mean PA in minutes according to cancer worry.

\begin{tabular}{|c|c|c|c|c|c|c|}
\hline Worry Levels & $\mathrm{N}$ & Mean PA Minutes & SD & $\mathrm{df}$ & $x^{2}$ & $\mathrm{p}$ \\
\hline Not at all & 542 & 20.0 & 16.7 & & & \\
\hline Slightly & 852 & 21.6 & 17.6 & & & \\
\hline Somewhat & 836 & 22.7 & 18.5 & 4 & 13.708 & .008 \\
\hline Moderately & 332 & 19.3 & 15.6 & & & \\
\hline Extremely & 144 & 20.8 & 18.6 & & & \\
\hline
\end{tabular}

why cancer worry is associated with PA behaviors and why elevated levels are linked to lower PA durations. This may be related to negative arousal and amplification of threat appraisals, as described by Aspinwall and Taylor (1997), which may shut down proactive coping efforts. Additional research is essential, specifically longitudinal research, to better understand these associations. Despite these limitations, using a large nationally representative sample may exceed the limitations as the results support generalizability.

\section{CONCLUSION}

The current study showed some support for previous literature suggesting that cancer worry is associated with health-promoting behaviors. Results indicated that while a certain level of cancer worry can decrease physical activity among adults, other levels of worry may increase it. Thus, these findings may inform psychosocial and health promotion interventions and future research. Further research is necessary to assess the causal nature of the association between cancer worry and physical activities and whether this association is observed in other health-promoting behaviors like nutrition intake. Additionally, future research may elucidate underlying psychological factors behind this relationship.

\section{Financial Support}

The first author was supported by a William F. and Margaret W. Scandling Scholar Award from the Warner School of Education and Human Development at the University of Rochester.

Competing interests

None declared.

\section{References}

Andersen, M. R., Smith, R., Meischke, H., Bowen, D., \& Urban, N. (2003). Breast cancer worry and mammography use by women with and without a family history in a population-based sample. Cancer Epidemiology and Prevention Biomarkers, 12(4), 314-320.

Aspinwall, L. G., \& Taylor, S. E. (1997). A stitch in time: Self-regulation and proactive coping. Psychological Bulletin, 121(3), 417-436. https://doi. org/10.1037/0033-2909.121.3.417

Behrens, G., \& Leitzmann, M. F. (2013). The association between physical activity and renal cancer: systematic review and meta-analysis. British Journal of Cancer, 108(4), 798-811. https://doi. org/10.1038/bjc. 2013.37

Bernat, J. K., Anderson, L. B., Parrish-Sprowl, J., \& Sparks, G. G. (2015). Exploring the association between dispositional cancer worry, perceived risk, and physical activity among college women. Journal of American College Health, 63(3), 216220. https://doi.org/10.1080/07448481.2014.98 3927

Brown, J. C., Winters-Stone, K., Lee, A., \& Schmitz, K. H. (2012). Cancer, physical activity, and exercise. In R. Terjung (Ed.), Comprehensive Physiology (p. c120005). John Wiley \& Sons, Inc. https://doi.org/10.1002/cphy.c120005

Brunet, J., Amireault, S., Chaiton, M., \& Sabiston, C. M. (2014). Identification and prediction of physical activity trajectories in women treated for breast cancer. Annals of Epidemiology, 24(11), 837-842. https://doi.org/10.1016/j. annepidem.2014.07.004

Clancy, F., O’Connor, D. B., \& Prestwich, A. (2020). Do worry and brooding predict health behaviors? A daily diary investigation. International Journal of Behavioral Medicine, 27, 591-601.

Consedine, N. S., Magai, C., Krivoshekova, Y. S., Ryzewicz, L., \& Neugut, A. I. (2004a). Fear, anxiety, worry, and breast cancer screening behavior: a critical review. Cancer Epidemiology, Biomarkers and Prevention, 13(4), 501-510.

Consedine, N. S., Magai, C., \& Neugut, A. I. (2004b). The contribution of emotional characteristics to breast cancer screening among women from six ethnic groups. Preventive Medicine, 38(1), 64-77. https://doi.org/10.1016/j. ypmed.2003.09.030

Davey, G. C., Jubb, M., \& Cameron, C. (1996). Catastrophic worrying as a function of changes in problem-solving confidence. Cognitive Therapy and Research, 20(4), 333-344.

Dunn, O. J. (1964). Multiple comparisons using rank sums. Technometrics, 6, 241-252. https:// doi.org/10.1080/00401706.1964.10490181

Durazo, A., \& Cameron, L. D. (2019). Representa- 
tions of cancer recurrence risk, recurrence worry, and health-protective behaviours: An elaborated, systematic review. Health Psychology Review, 13(4), 447-476. https://doi.org/10.1080/174371 99.2019 .1618725

Eliassen, A. H., Hankinson, S. E., Rosner, B., Holmes, M. D., \& Willett, W. C. (2010). Physical activity and risk of breast cancer among postmenopausal women. Archives of Internal Medicine, 170(19), 1758-1764. https://doi.org/10.1001/ archinternmed.2010.363

Ferrer, R. A., Portnoy, D. B., \& Klein, W. M. (2013). Worry and risk perceptions as independent and interacting predictors of health protective behaviors. Journal of Health Communication, 18(4), 397-409. https://doi.org/10.1080/10810730.201 2.727954

Finney Rutten, L. J., Blake, K. D., Skolnick, V. G., Davis, T., Moser, R. P., \& Hesse, B. W. (2020). Data resource profile: The national cancer institute's health information national trends survey (HINTS). International Journal of Epidemiology, 49(1), 17-17j.

Hay, J. L., Buckley, T. R., \& Ostroff, J. S. (2005). The role of cancer worry in cancer screening: a theoretical and empirical review of the literature. Psycho-Oncology: Journal of the Psychological, Social and Behavioral Dimensions of Cancer, 14(7), 517-534. https://doi.org/10.1002/pon.864

Hay, J. L., McCaul, K. D., \& Magnan, R. E. (2006). Does worry about breast cancer predict screening behaviors? A meta-analysis of the prospective evidence. Preventive Medicine, 42(6), 401-408. https://doi.org/10.1016/j.ypmed.2006.03.002

Janssen, E., Waters, E. A., Van Osch, L., Lechner, L., \& De Vries, H. (2014). The importance of affectively-laden beliefs about health risks: The case of tobacco use and sun protection. Journal of Behavioral Medicine, 37(1), 11-21. doi:10.1007/ s10865-012-9462-9

Keimling, M., Behrens, G., Schmid, D., Jochem, C., \& Leitzmann, M. F. (2014). The association between physical activity and bladder cancer: systematic review and meta-analysis. British Journal of Cancer, 110(7), 1862-1870. https://doi. org/10.1038/bjc.2014.77

Klein, W. M., Zajac, L. E., \& Monin, M. M. (2009). Worry as a moderator of the association between risk perceptions and quitting intentions in young adult and adult smokers. Annals of Behavioral Medicine, 38(3), 256-261. https://doi. org/10.1007/s12160-009-9143-2

Kossman, D. A., Williams, N. I., Domchek, S. M., Kurzer, M. S., Stopfer, J. E., \& Schmitz, K. H. (2011). Exercise lowers estrogen and progesterone levels in premenopausal women at high risk of breast cancer. Journal of Applied Physiology, 111(6), 1687-1693. https://doi.org/10.1152/ japplphysiol.00319.2011

Liu, L., Shi, Y., Li, T., Qin, Q., Yin, J., Pang, S., ... \& Wei, S. (2016). Leisure time physical activity and cancer risk: evaluation of the WHO's recommendation based on 126 high-quality epidemiological studies. British Journal of Sports Medi- cine, 50(6), 372-378. http://dx.doi.org/10.1136/ bjsports-2015-094728

Lumish, H. S., Steinfeld, H., Koval, C., Russo, D., Levinson, E., Wynn, J., ... \& Chung, W. K. (2017). Impact of panel gene testing for hereditary breast and ovarian cancer on patients. Journal of Genetic Counseling, 26(5), 1116-1129. https://doi.org/10.1007/s10897-017-0090-y

Miles, A., Voorwinden, S., Chapman, S., \& Wardle, J. (2008). Psychologic predictors of cancer information avoidance among older adults: the role of cancer fear and fatalism. Cancer Epidemiology and Prevention Biomarkers, 17(8), 1872-1879. https://doi.org/10.1158/1055-9965.EPI-08-0074

Moore, S. C., Lee, I. M., Weiderpass, E., Campbell, P. T., Sampson, J. N., Kitahara, C. M., ... \& Adami, H. O. (2016). Association of leisure-time physical activity with risk of 26 types of cancer in 1.44 million adults. JAMA Internal Medicine, 176(6), 816-825. https://doi.org/10.1001/ jamainternmed.2016.1548

Moser, R. P., Mccaul, K., Peters, E., Nelson, W., $\&$ Marcus, S. E. (2007). Associations of perceived risk and worry with cancer health-protective actions: data from the Health Information National Trends Survey (HINTS). Journal of Health Psychology, 12(1), 53-65. https://doi. org/10.1177\%2F1359105307071735

Ogle, D. H. (2016). Introductory fisheries analyses with R (Vol. 32). CRC Press.

Palmero, E. I., Campacci, N., Schüler-Faccini, L., Giugliani, R., Rocha, J. C. C. D., Vargas, F. R., \& Ashton-Prolla, P. (2020). Cancer-related worry and risk perception in Brazilian individuals seeking genetic counseling for hereditary breast cancer. Genetics and Molecular Biology, 43(2).

Persoskie, A., Ferrer, R. A., \& Klein, W. M. (2014). Association of cancer worry and perceived risk with doctor avoidance: an analysis of information avoidance in a nationally representative US sample. Journal of Behavioral Medicine, 37(5), 977-987. https://doi.org/10.1007/s10865-013-9537-2

Pizot, C., Boniol, M., Mullie, P., Koechlin, A., Boniol, M., Boyle, P., \& Autier, P. (2016). Physical activity, hormone replacement therapy and breast cancer risk: A meta-analysis of prospective studies. European Journal of Cancer, 52, 138-154. https:// doi.org/10.1016/j.ejca.2015.10.063

Psaltopoulou, T., Ntanasis-Stathopoulos, I., Tzanninis, I. G., Kantzanou, M., Georgiadou, D., \& Sergentanis, T. N. (2016). Physical activity and gastric cancer risk: a systematic review and meta-analysis. Clinical Journal of Sport Medicine, 26(6), 445-464. https://doi.org/10.1097/ JSM.0000000000000316

Quaife, S. L., Waller, J., von Wagner, C., \& Vrinten, C. (2019). Cancer worries and uptake of breast, cervical, and colorectal cancer screening: a population-based survey in England. Journal of Medical Screening, 26(1), 3-10. https://doi. org/10.1177\%2F0969141318796258

Vrinten, C., Waller, J., von Wagner, C., \& Wardle, J. (2015). Cancer fear: facilitator and deterrent to participation in colorectal cancer screening. Can- 
cer Epidemiology, Biomarkers and Prevention, 24(2), 400-405. https://doi.org/10.1158/10559965.EPI-14-0967

Wickham, H., Francois, R., Henry, L., \& Müller, K. (2015). dplyr: A Grammar of Data Manipulation. R package version 0.4. 3. R Found. Stat. Comput., Vienna. https://CRAN. R-project. org/ package $=$ dplyr.

Yong, H. H., Borland, R., Thrasher, J. F., Thompson, M. E., Nagelhout, G. E., Fong, G. T., ... \& Cummings, K. M. (2014). Mediational pathways of the impact of cigarette warning labels on quit attempts. Health Psychology, 33(11), 1410. doi: $10.1037 /$ hea0000056 